\title{
The QT interval in atrial fibrillation
}

\author{
G R PAI, J M RAWLES \\ From the Department of Medicine and Therapeutics, University of Aberdeen
}

SUMMARY The electrocardiogram was recorded for 100 seconds in 50 patients with atrial fibrillation to determine the relations between QT intervals and both the mean and instantaneous ventricular rates. The mean ventricular rate was 94 beats per minute with a mean QT interval of $\vec{\omega}$ $357 \mathrm{~ms}$. The mean QTc, corrected beat by beat with Bazett's formula, was 444 ms-longer than reported for sinus rhythm. Between subjects, the mean QT interval was linearly related to the mean $R R$ interval, with a slope of $+21 \%$. Within all 50 recordings there was a statistically significant correlation between QT intervals and immediately preceding $R R$ intervals, with an average slope $\sigma$ of $+7 \%$. This within subject $Q T / R R$ interval slope was greater at faster mean ventricular rates. In $\vec{\circ}$ atrial fibrillation, as in sinus rhythm, the $\mathrm{QT}$ interval is a function of both the mean ventricular rate 을 and the instantaneous ventricular rate, with the mean ventricular rate predominating; a simple correction of QT intervals for heart rate is therefore inadequate. Comparison of uncorrected QT $\subseteq$ intervals with those of earlier published series of people in sinus rhythm, however, suggested that $\overparen{\odot}$ atrial fibrillation is associated with prolongation of the mean QT interval.

The duration of the QT interval of the electrocardiogram is a function of the heart rate; it is positively correlated with preceding $R R$ intervals. Before a QT interval can be assessed in sinus rhythm, its duration must be adjusted for the mean $R R$ interval or heart rate. For this purpose various formulas have been used that imply a linear relation between the QT interval and the $R R$ interval (Schlamowitz, ${ }^{1}$ Simonson et al $^{2}$ ), or its square root $\left(\right.$ Bazett $\left.^{3}\right)$, cube root (Fridericia ${ }^{4}$ ), or logarithm $\left(\right.$ Ashman ${ }^{5}$.

It has recently been shown, however, that the duration of the QT interval depends not only on the prevailing heart rate but also on the instantaneous interval between beats. ${ }^{6}$ When the paced heart rate was suddenly increased and then maintained, there was an immediate shortening of the QT interval followed by a further more gradual shortening over several minutes. These observations imply that a simple correction of QT interval for heart rate is inadequate.

Atrial fibrillation is characterised by considerable beat to beat changes of heart rate, with a wide range of mean heart rates in different subjects. It therefore provides an opportunity to examine the QT interval

Requests for reprints to Dr J M Rawles, Department of Medicine and Therapeutics, University of Aberdeen, Foresterhill, Aberdeen AB9 2ZD.

Accepted for publication 14 February 1989 as a function of both the instantaneous and the mean heart rate. We examined the relations between QT and $R R$ intervals within and between 50 patients with atrial fibrillation.

\section{Patients and methods}

\section{PATIENTS}

We studied 50 patients ( 19 men and 31 women; aged 31-87 (mean 70)) with established atrial fibrillation. The causes of atrial fibrillation were: idiopathic, 26 ; ischaemic heart disease, nine; valvar heart disease, six; pulmonary disease, six; and thyrotoxicosis, three. Thirty one patients were taking digoxin; none $ᄋ$ was taking other drugs known to affect the QT interval.

\section{ELECTROCARDIOGRAM RECORDINGS AND MEASUREMEN T}

Patients lay down for 15 minutes before an average of 151 beats were recorded by a standard lead I at a $N$ paper speed of $25 \mathrm{~mm} /$ second. The $Q T$ and $R R \hat{\omega}$ intervals were measured with a digitising pad and $\sigma$ microcomputer with a resolution of $0.1 \mathrm{~mm}, 0$ equivalent to $4 \mathrm{~ms}$; the results were stored on disc. $\mathbb{\Phi}$ The average $(\mathrm{SD})$ difference between replicated $\stackrel{\oplus}{?}$ measurements of $213 \mathrm{RR}$ intervals was $0.20 \mathrm{~T}$ (10.8) $\mathrm{ms}$.

STATISTICAL ANALYSIS

We used Bazett's equation ${ }^{3}$ to correct QT intervals 
for heart rate: $Q T c=Q T /(R R)^{1 / 2}$, where $R R$ is in seconds. It was used in two ways: either each $\mathrm{QT}$ interval was corrected by the preceding $R R$ interval and the mean corrected QT interval of all beats was calculated, or we corrected the mean QT interval for each patient using the mean $R R$ interval.

Linear and multiple regression was carried out to study the relations between $Q T$ and $R R$ intervals, and paired and unpaired $t$ tests were used as appropriate to compare means.

\section{Results}

MEAN RR, QT, AND QTC INTERVALS

The table shows the averages of mean heart rate, $R R$, QT, and QTc intervals. None of these measurements was significantly different in men or women, in those taking or not taking digoxin, or in those aged over 69 or under 70.

Correction of QT intervals beat by beat gave significantly higher values than the mean $R R$ intervals $(\mathrm{p}<0.001)$. Of 50 patients, 28 had a mean QTc $>\mathbf{4 4 0}$ ms corrected beat by beat compared with 22 when corrected by $R R$ interval means. There was no relation between a prolonged QTc and diagnosis.

Figure 1 shows the distribution of mean $R R$ and QT intervals in our 50 patients with atrial fibrillation in relation to the normal range in 6000 cases in sinus rhythm (data of Lepeshkin, redrawn from Ahnve ${ }^{7}$ ). Most cases fall in the upper half of the normal distribution but in eight cases the mean QT interval is excessive for the mean $R R$ interval.

\section{RELATION BETWEEN MEAN QT AND MEAN RR}

INTERVALS BETWEEN PATIENTS

The mean QT and $R R$ intervals in 50 subjects were positively correlated $(r=0.77, p<0.001)$, and the regression equation was $Q T=213+0.21(R R)$, where both the QT and RR intervals are expressed in $\mathrm{ms}$ (fig 1). The regression lines for the data of Schlamowitz, ${ }^{1}$ Simonson et $a l_{,}{ }^{2}$ and Ahnve $^{7}$ are shown for comparison.

The slope of the regression line for the relation between $Q T$ and $R R$ intervals in atrial fibrillation was not significantly different in men or women, in

Table Averages (SD) of mean heart rate, $R R, Q T$, and $Q T$ c intervals corrected beat by beat or by mean $R R$ in 50 patients with atrial fibrillation

\begin{tabular}{lc}
\hline Variables (beats/min) & Mean (SD) \\
\hline Heart rate & $94.0(26 \cdot 5)$ \\
RR intervals (ms) & $683.6(173 \cdot 1)$ \\
QT intervals (ms) & $357.6(47 \cdot 7)$ \\
QTc (beat) intervals (ms) & $443.7(38.6)$ \\
QTc (mean) intervals (ms) & $437.4(37 \cdot 7)$ \\
\hline
\end{tabular}

those taking or not taking digoxin, or in those aged 69 or under 70. Inspection of the plot shows no obvious non-linearity, but the correlation coefficient was slightly increased, from 0.77 to 0.78 , when $R R^{1 / 2}$ was used in place of mean RR.

\section{RELATION BETWEEN QT AND RR INTERVALS WITHIN PATIENTS}

In every case, $Q T$ intervals were positively correlated with immediately preceding $R R$ intervals $(p<0.001$ in $41 ; p<0.01$ in six; $p<0.05$ in three); the average of the correlation coefficients was $0 \cdot 44$. Only in eight cases were QT intervals significantly correlated with pre-preceding $R R$ intervals as well (six negative, two positive).

In fig 2 the slopes of the individual QT/RR interval relations are plotted against mean $R R$ intervals. The average slope was $7 \%$, and the shorter the mean $R R$ interval, the higher the heart rate, the greater the slope, and the greater the influence of the preceding $R R$ interval on the QT interval. However, the average slope within individuals was only a third of that between individuals, and every within individual slope was less than that between individuals (fig 3 ).

Figure 4 shows a plot of QT and RR intervals in a 76 year old woman with a mean ventricular rate of 76 . In this case the relation is better described as quadratic than linear. In 31 cases a quadratic equation gave a slightly better fit, and the average multiple correlation coefficient for the quadratic equation was 0.45 compared with 0.44 for linear regression.

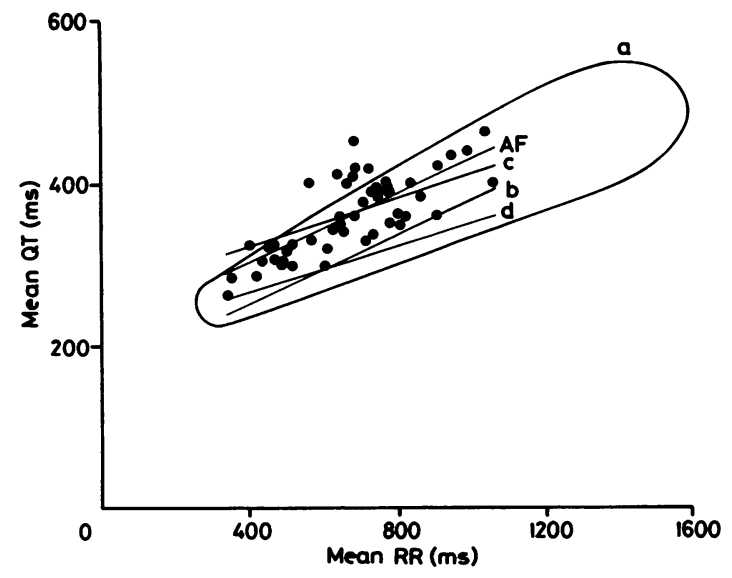

Fig 1 Scatter plot and regression line of mean $Q T$ and $R R$ intervals in 50 patients with atrial fibrillation $(\boldsymbol{A F})$ in. relation to (a) the range of values in 6000 healthy subjects (Lepeshkin ${ }^{8}$ ), (b) the regression line from 495 healthy subjects (Schlamowitz ${ }^{1}$ ), (c) the regression line from 960 healthy subjects (Simonson et $a^{2}$ ), and $(d)$ the regression line from 152 patients with ischaemic heart disease on digitalis $\left(A h n v e^{7}\right)$. 


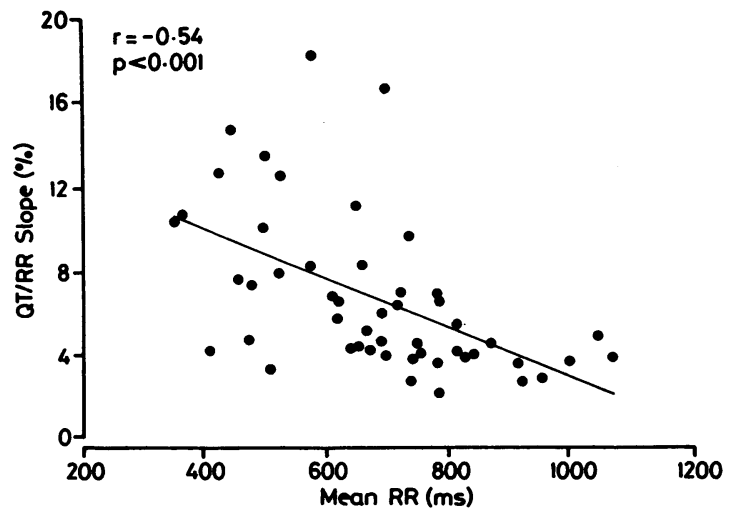

Fig 2 Scatter plot and regression line of the within subject $Q T / R R$ interval slope and the mean $R R$ interval in 50 patients with atrial fibrillation.

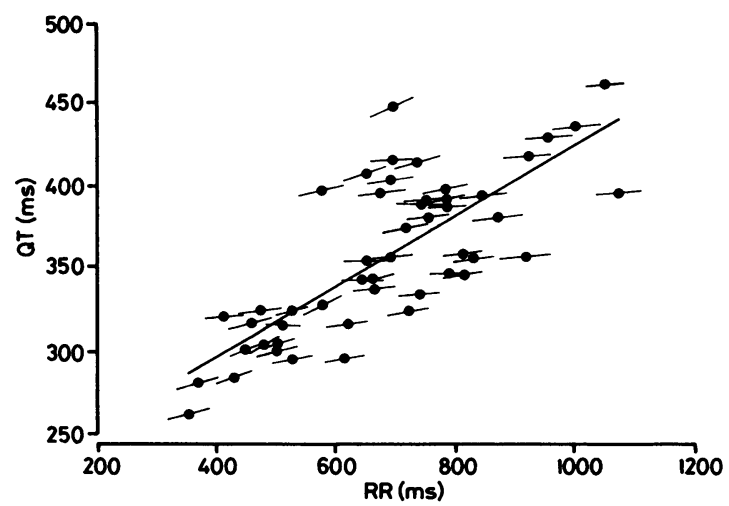

Fig 3 Regression lines for the between subject and within subject $Q T / R R$ interval relations in 50 patients with atrial fibrillation.

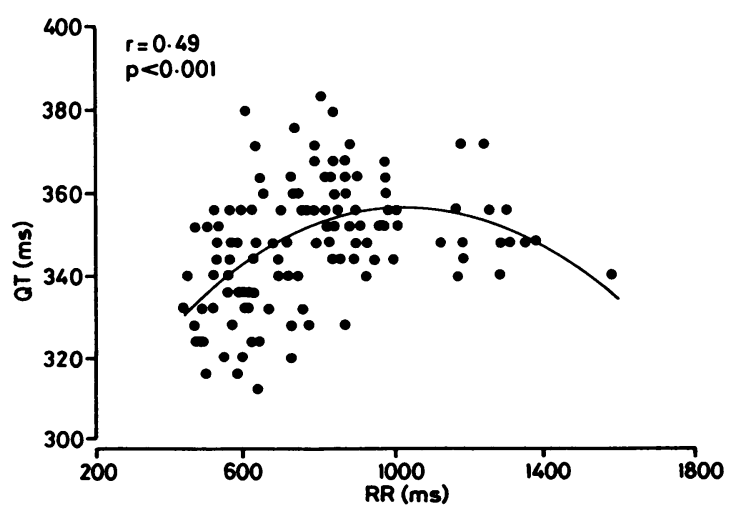

Fig $4 Q T$ and $R R$ intervals and the best fit quadratic regression line in a 76 year old woman with atrial fibrillation.

\section{Discussion}

The QT interval of the surface electrocardiogram corresponds to electrical systole, during which $\stackrel{5}{\leftrightarrows}$ ventricular muscle is depolarised, as may be shown $\bar{O}$ by unipolar electrodes placed within the heart. If $\bar{F}$ there is a regular ventricular rate and an extra $\frac{\bar{\omega}}{7}$ stimulus is applied immediately after depolarisation $\mathbb{\unrhd}$ there is no response, and the ventricle is said to be refractory. If the stimulus is applied later then depolarisation occurs, but its duration depends on the interval between the previous beat and the stimulus. The curve depicting the relation between the delay in the stimulus and the duration of depolarisation is called the restitution curve. The $\rightarrow$ shape and position of the restitution curve are $\dot{\sigma}$ affected by the basic ventricular rate, being depressed $O T$ at higher heart rates. A change of ventricular rate 0 produces an immediate change in the duration of $\frac{9}{?}$ depolarisation and the QT interval followed by a more gradual change over the next few minutes. ${ }^{6}$

In atrial fibrillation each beat may be considered as $\overparen{D}$ a depolarising stimulus, occurring a variable time $\vec{\oplus}$ after the preceding beat. The duration of the sub- $\infty$ sequent QT interval will vary according to the restitution curve at that particular mean ventricular rate. The within subject $Q T / R R$ interval relation corresponds to the restitution curve except that, in contrast with the experimental situation and with sinus rhythm, the rhythm of ventricular contraction is irregular. In atrial fibrillation, therefore, both instantaneous and mean ventricular rates would be expected to determine the duration of the QT interval: this is what we showed. The influence of the mean ventricular rate exceeded that of the instantaneous ventricular rate, the slope of the $Q T / R R$ interval relation between subjects being $21 \%$, compared with an average of $7 \%$ within subjects. An increasing mean ventricular rate altered both the position and the slope of the within subject QT/RR interval relation; it depressed the position and $\frac{9}{9}$ increased the slope.

IS THE QT INTERVAL IN ATRIAL FIBRILLATION DIFFERENT FROM THAT IN SINUS RHYTHM?

The occurrence of atrial fibrillation implies some $N$ underlying cardiac abnormality which was overt in $\mathrm{W}$ many of our cases. It is therefore difficult to be sure $\bar{\sigma}$ whether any differences from normal of QT intervals $O$ are due to the arrhythmia, or the pathological process $\Phi$ that caused it. Ischaemia and inflammation are important causes of QT prolongation, ${ }^{8}$ but no patient ${ }^{7}$ was known to have any cardiac inflammatory process, $\stackrel{\circ}{\mathbb{D}}$ and none of the patients with ischaemic heart disease had ischaemia at rest or had recently had a myo- $\mathbb{Q}$ cardial infarction. A second difficulty is that of 
correction for heart rate.

In atrial fibrillation the relation between heart rate and QT interval is complex and not readily encompassed by a simple equation such as those proposed for sinus rhythm. It is therefore inappropriate to correct QT intervals with a formula such as Bazett's. When we used Bazett's formula on a beat to beat basis 28 of 50 patients had a mean QTc above the upper limit of normal of $440 \mathrm{~ms}$; whereas a scatter plot of mean uncorrected QT and RR intervals showed only eight definitely abnormal results compared with Lepeschkin's results in 6000 healthy subjects with sinus rhythm ${ }^{8}$ (fig 1). Schlamowitz carried out linear regression of QT against $R R$ intervals in $\mathbf{4 9 5}$ people in sinus rhythm. ${ }^{1}$ The correlation coefficient of 0.78 was similar to the between patient correlation in our study of patients with atrial fibrillation. His regression line seems to fit the data of Lepeshkin very well, but is parallel to and below our regression line for patients with atrial fibrillation. The regression line for Simonson et al's data from 960 healthy people has a lower slope and crosses our own at its midpoint. ${ }^{2}$ Ahnve described the regression line from 152 patients with ischaemic heart disease on digitalis, and that line too lay below our own. ${ }^{7}$

We tentatively conclude that in atrial fibrillation uncorrected QT intervals are generally longer than they are at the same ventricular rate in sinus rhythm, but only a few mean QT intervals fall outside the wide range encountered in the largest published series. Comparison with data from patients with ischaemic heart disease suggests that the prolongation of QT intervals in atrial fibrillation may result from the arrhythmia rather than any underlying abnormality.

G R Pai was supported by a research grant from the Merrell Dow Research Institute. A research grant from Grampian Health Board is gratefully acknowledged.

\section{References}

1 Schlamowitz I. An analysis of the time relationships within the cardiac cycle in electrocardiograms of normal men. $I$. The duration of the $Q-T$ interval and its relationship to the cycle length ( $R-R$ interval). Am Heart $J$ 1946;31:329-42.

2 Simonson E, Cady LD, Woodbury M. The normal Q-T interval. Am Heart J 1962;63:747-53.

3 Bazett HC. An analysis of the time-relations of electrocardiograms. Heart 1920;7:353-70.

4 Fridericia LS. Die Systolendauer im Elektrokardiogramm bei normalen Menshen und bei Herzkranken. Acta Med Scand 1920;53:469-86.

5 Ashman $R$. The normal duration of the Q-T interval. Am Heart J 1942;23:522-34.

6 Seed WA, Noble MIM, Oldershaw P, et al. Relation of human cardiac action potential duration to the interval between beats: implications for the validity of rate corrected QT interval (QTc). Br Heart $J$ 1987;57:32-7.

7 Ahnve S. Correction of the QT interval for heart rate: review of different formulas and the use of Bazett's formula in myocardial infarction. Am Heart $J$ 1985;109:568-74.

8 Lepeschkin E. Modern electrocardiography, vol 1. Baltimore: The Williams and Wilkins Co, 1951: $180-8$. 